Volume 40

November 2010
Issue 6

ISSN 0169-8141

This Issue Completes Volume 40

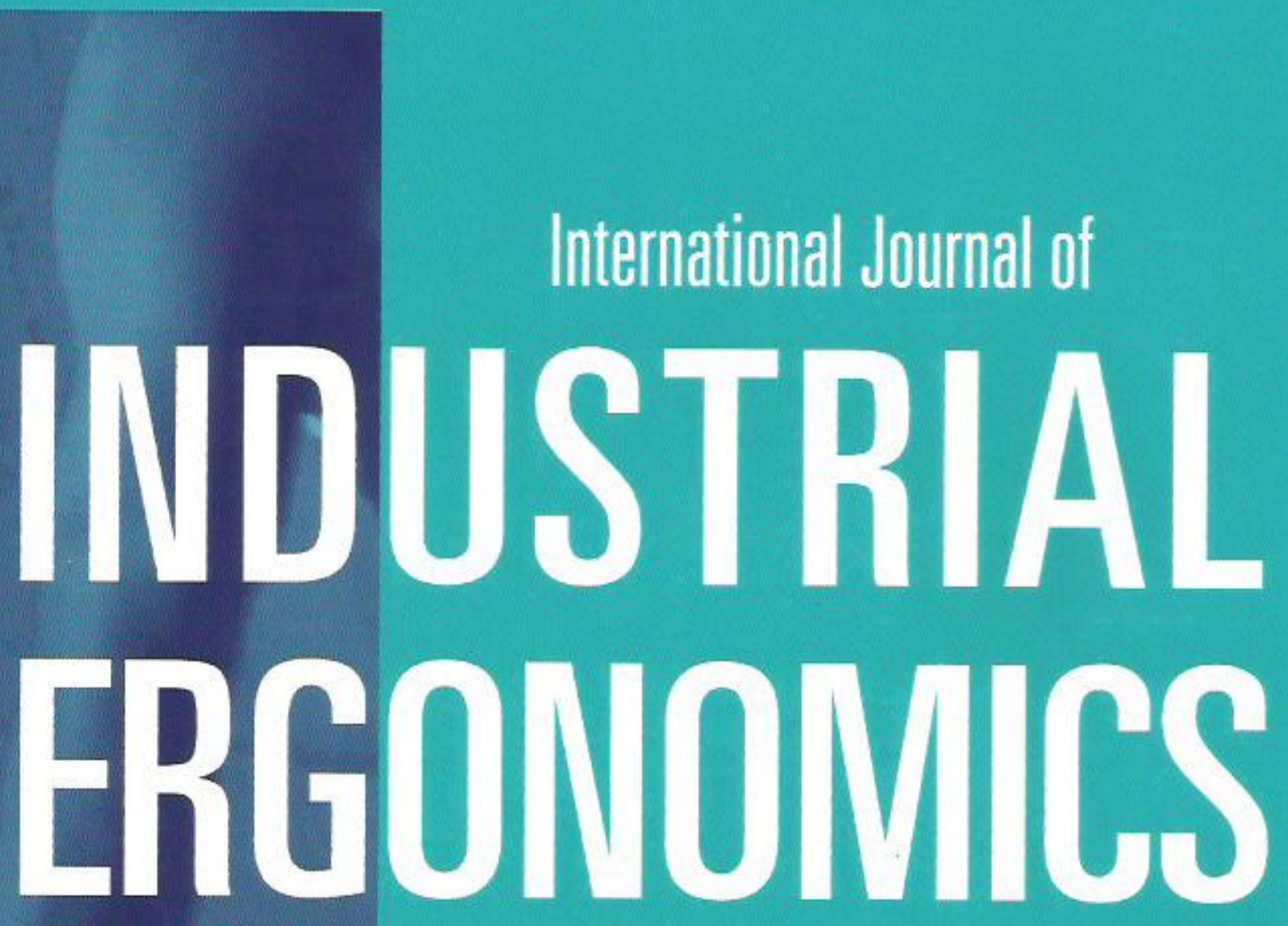

In collaboration with IEA and ISOES 


\section{International Journal of Industrial Ergonomics \\ Volume 40, Issue 6, November 2010}

\section{Contents}

C. Müller, L. Tomatis, T. Läubli

Muscular load and performance compared between a pen and a computer mouse as input devices

L. Rusli, A. Luscher, C. Sommerich

Force and tactile feedback in preloaded cantilever snap-fits under manual assembly

S. Asensio-Cuesta, J.A. Diego-Mas, J. Alcaide-Marzal

Applying generalised feedforward neural networks to classifying industrial jobs in terms of risk of low back disorders

H.S.J. Rashid, C.S. Place, G.R. Braithwaite

Helicopter maintenance error analysis: Beyond the third order of the HFACS-ME

C.A. Duncan, S.N. MacKinnon, W.J. Albert

Changes in thoracolumbar kinematics and centre of pressure when performing stationary tasks in moving environments

Y.-L. Lee

Comparison of the conventional point-based and a proposed finger probe-based touch screen interaction techniques in a target selection task

M. Canjuga, T. Läubli, G.F. Bauer

Can the job demand control model explain back and neck pain? Cross-sectional study in a representative sample of Swiss working population

H. Kim

Effective organization of design guidelines reflecting designer's design strategies

A.H.S. Chan, A.W.Y. Ng

Investigation of guessability of industrial safety signs: effects of prospective-user factors and cognitive sign features

R. Mani, S. Milosavljevic, S.J. Sullivan

The effect of occupational whole-body vibration on standing balance: A systematic review

S. Rakheja, R.G. Dong, S. Patra, P.E. Boileau, P. Marcotte, C. Warren

Biodynamics of the human body under whole-body vibration: Synthesis of the reported data

S. Kwon, C. Kim, S. Kim, S.H. Han

Two-mode target selection: Considering target layouts in small touch screen devices

Y.S. Park, S.H. Han

One-handed thumb interaction of mobile devices from the input accuracy perspective

T.K. Chuan, M. Hartono, N. Kumar

Anthropometry of the Singaporean and Indonesian populations

D.T. Goomas, P.H.P. Yeow

Ergonomics improvement in a harsh environment using an audio feedback system

News

November 2010

Articles are abstracted/indexed in: CAD/CAM Abstracts, Cambridge Scientific Abstracts, Current Contents: Engineering, Computing and Technology, Current Contents: Social \& Behavioral Sciences, EMBASE, Engineering Index/ Compendex, Ergonomics Abstracts, NIOSHTIC, Research Alert, Science Citation Index, SciSearch, Social Sciences Citation Index. Also covered in the abstract and citation database SCOPUS ${ }^{\circledR}$. Full text available on ScienceDirect ${ }^{\circledR}$.

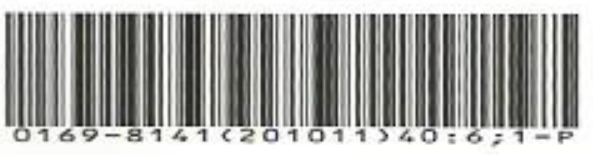




\title{
Anthropometry of the Singaporean and Indonesian populations
}

\author{
Tan Kay Chuan*, Markus Hartono, Naresh Kumar \\ Department of Industrial and Systems Engineering, National University of Singapore, Singapore
}

\section{A R R I I C L E I N F}

\section{Article history:}

Received 16 December 2009

Received in revised form

10 March 2010

Accepted 3 May 2010

Available online 26 May 2010

\section{Keywords:}

Anthropometry

Body mass index (BMI)

Singaporean

Indonesian

\begin{abstract}
A B S T R A C T
This research collected anthropometric data of the Singaporean and Indonesian populations. The data were mainly from university students. In total, 245 male and 132 female subjects from Indonesia and 206 male and 109 female subjects from Singapore were measured. The Singapore data were divided into three sub-groups, comprising Singapore overall, Singapore citizens, and the Chinese ethnic sub-group. The Indonesians data were divided into two sub-groups, comprising Indonesia citizens and Indonesia Chinese. This study used 36 measurement dimensions. The authors made a comparison with previous anthropometric data collected in 1990 of over a thousand Singaporeans.

The main contributions of this study are: i) an updated anthropometric database of Singaporeans and Indonesians, ii) a comparison of the two samples obtained, and iii) a projection of dimensional changes over time from comparing past to more recent anthropometric data. Statistical analyses show that Singaporeans (both male and female) tend to have larger dimensions than Indonesians in general. In addition, the data reveal the current sample to be significantly larger on more than 50 percent of the dimensions measured, for both males and females.

In providing instances of possible application, the Body Mass Index (BMI) of all sub-groups was calculated. The results show both samples to have normal indexes with BMIs in the range of 18.5-25.0. This paper presents also an empirical estimation of unknown anthropometric characteristics using the Ratio Scaling Method. The purpose is to estimate uncollected anthropometric data based on a given scaling dimension. Overall, the reported anthropometric data and analyses can be used as relevant consideration in product and systems design.

Relevance to industry: The findings of this study indicate differences between Singaporean and Indonesian anthropometry in the citizen and Chinese sub-groups. The utilization of an updated anthropometric database that incorporates geographical origin and ethnic group is useful. Product designers would be able to cater to a wider range of target users.
\end{abstract}

(c) 2010 Elsevier B.V. All rights reserved.

\section{Introduction}

Products can be deemed successful only when people are able to use them well. This is in accordance with the fundamental principle of ergonomics which is to fit the task to the human (Kroemer and Grandjean, 1997). In doing so, a high level of safety and worker efficiency can be achieved. This means of user-centered design involves the product, the user, and the task. However, variation in body dimension among people, between the sexes, and among different races, can make product design problematic. While it is impossible to design systems to suit all body types and sizes, it is prudent to deal at least with the important dimensions. Thus, anthropometry should be taken into account. Anthropometric data

\footnotetext{
* Corresponding author. Tel.: +65 6516 3128; fax: +65 7771434.

E-mail address: isetankc@nus.edu.sg (T.K. Chuan).
}

are useful in achieving effective design for high performance and productivity (Klamklay et al., 2008). Nowadays, the collection of anthropometric had been conducted through a sophisticated technology (i.e. three-dimensional measurement) which even proposing an error detection procedure (Park et al., 2009). A lack of anthropometric consideration in equipment design may lead to work-related injuries such as musculoskeletal disorder.

There exists anthropometry data of several Asia Pacific populations. Examples include the hand anthropometry of Jordanian (Mandahawi et al., 2008), static anthropometry of Tehran University students (Mououdi, 1997), anthropometry of the elderly in Australia (Kothiyal and Tettey, 2000), anthropometry of Taiwanese women (Huang and You, 1994), anthropometry of Portuguese workers (Barroso et al., 2005), anthropometry of Turkish woman (Gonen et al., 1991), anthropometry of the Turkish population (İșeri and Arslan, 2009), anthropometry of the Thai population (Klamklay et al., 2008), anthropometry of 

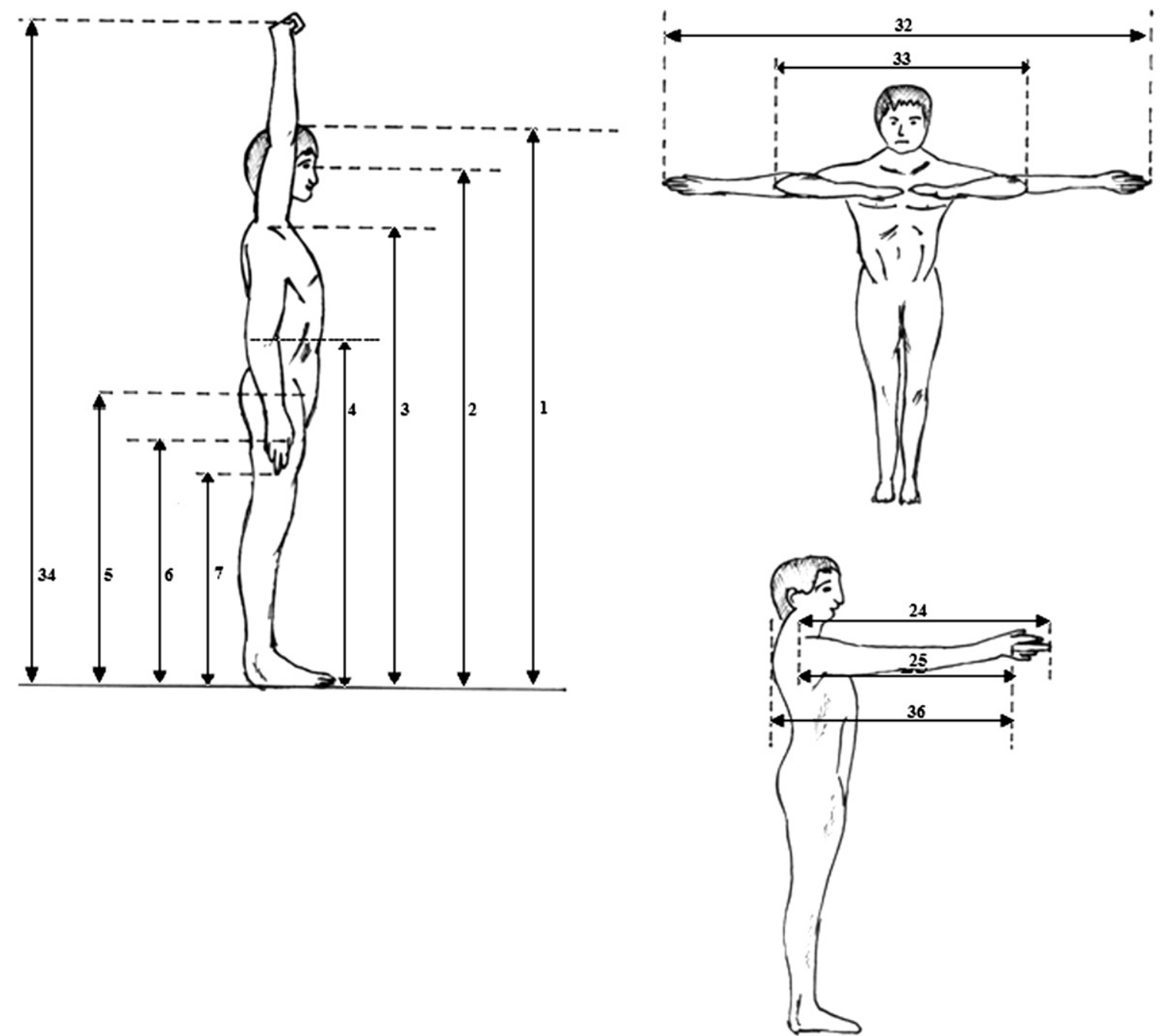

Fig. 1. Body measurement in standing position.

Bahraini school children (Al-Ansari and Mokdad, 2009), anthropometry of north eastern Indian female farm workers (Dewangan et al., 2008), and anthropometry of Sri Lankan university students (Thariq et al., 2010). Since 1990, however, there has been no publication of anthropometric data of the Singaporean population. This research updates the anthropometric data of Singaporeans. We analyze differences in data collected in 1990 and today. Inspired by Lin et al. (2004), we compared the anthropometric characteristics of two groups of people in South-east Asia. This study analyzed significant differences in body dimensions found between the Singaporean and Indonesian samples. Several sub-groups were covered, including population overall, citizens only, and an ethnic sub-group.

This research had four objectives. The first objective was to examine and compare the physical measurements of the Singaporean and Indonesian samples for all three sub-groups. The second objective was to analyze differences between the old and the new anthropometric data of the Singaporean samples. The third objective was to shed light on an important application of anthropometric data. The Body Mass Index (BMI) is taken as an illustrative application that can describe the health of Singaporeans and Indonesians. The final objective was to conduct an empirical estimation of unknown anthropometric characteristics using the Ratio Scaling Method as pioneered by Pheasant (1982).

\section{Method}

This research is a combination of two anthropometric data gathering exercises as mentioned earlier. The study and data gathering were carried out over a period of 36 months.

\subsection{Subjects}

Male and female students from a university in Singapore and a university in Indonesia voluntarily participated in this study. In total, 245 male and 132 female subjects from Indonesia (total of 377 subjects) and 206 male and 109 female subjects from Singapore (total of 315 subjects) with ages ranged from 18 to 45 years, were measured (grand total is 692 subjects).

In Indonesia, there were 245 males and 132 females in citizen sub-group and 88 males and 54 females in Chinese sub-group; whereas in Singapore, there were 206 males and 109 females in overall sub-group, 138 males and 57 females in citizen sub-group, and 104 males and 47 females in Chinese sub-group. For Indonesian population, the percentage of Chinese within the citizen sub-group was $37.67 \%$. This was also the same percentage of Chinese within the whole group since there were only 2 sub-groups for Indonesian subjects, i.e. citizen and Chinese. But for Singaporean population, the percentages of Chinese within the citizen sub-group and the 

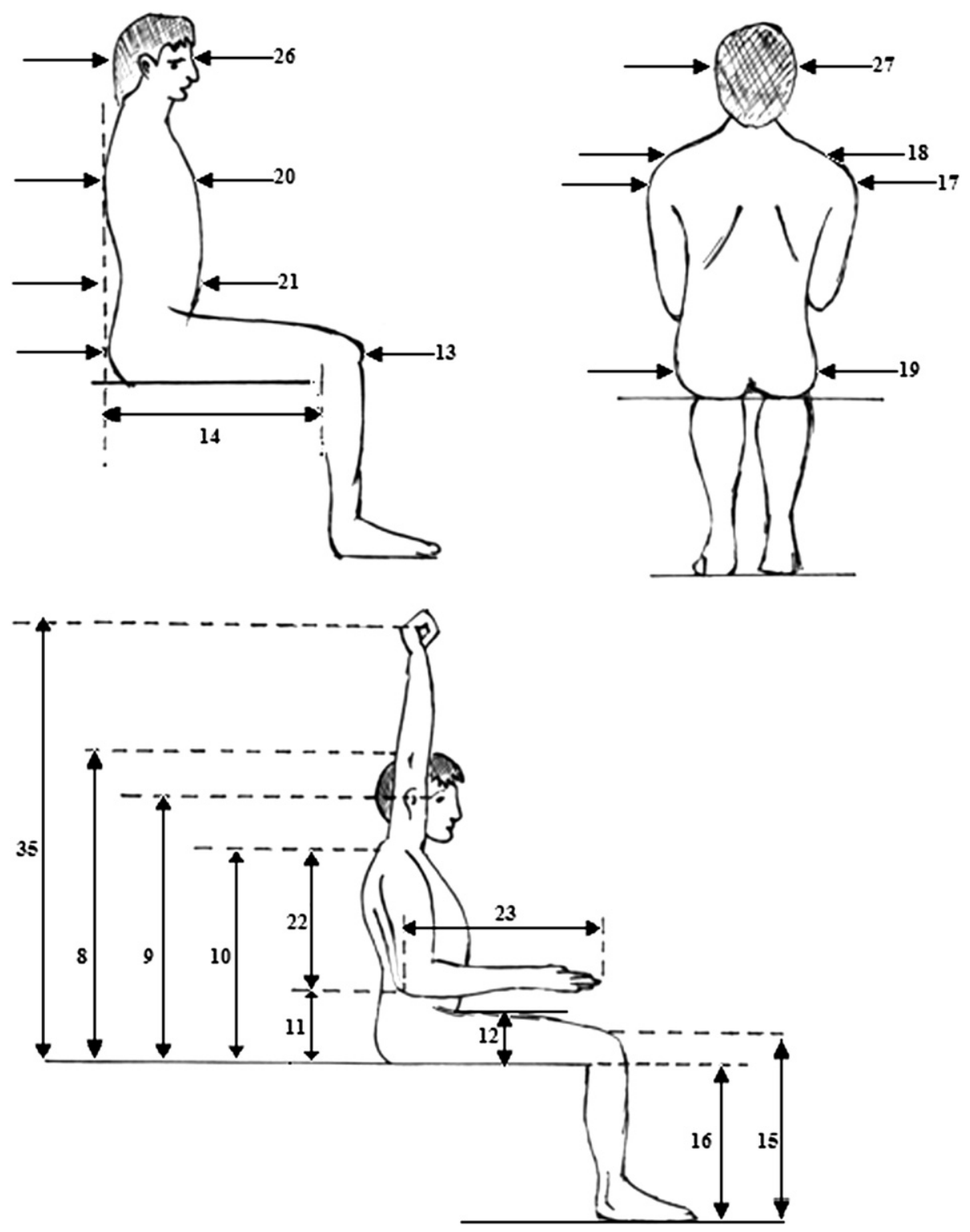

Fig. 2. Body measurement in sitting position.

whole group were $77.44 \%$ and $47.94 \%$ respectively. Most have taken a course in ergonomics/human factors engineering. Each received a token of appreciation for their time. It is assumed that university students are representative of a population's anthropometry.

The taking of anthropometric data is a sensitive issue to some people who may refuse to be measured. Due to this, we used convenience sampling as opposed to random sampling. Convenience sampling selects subjects arbitrarily and in an unstructured manner from the sample frame (Lohr, 1999).

\subsection{Body dimensions}

All measurements were taken in the afternoon. Subjects were barefooted, wearing t-shirts or shirts, and shorts or pants while the measurements were taken. For the anthropometric data of Indonesians, the body weight and 35 body dimensions were measured. For the anthropometry of Singaporeans, the body weight and 36 body dimensions were taken. The landmarks of body dimensions are shown in Figs. 1-3. The body dimensions and measurement as specified by Pheasant and Haslegrave (2006) were used.

\subsection{Equipment}

Traditional anthropometric tools were used rather than a more sophisticated and high-tech equipment such as a three-dimensional scanner. They are simple, portable, inexpensive, and as
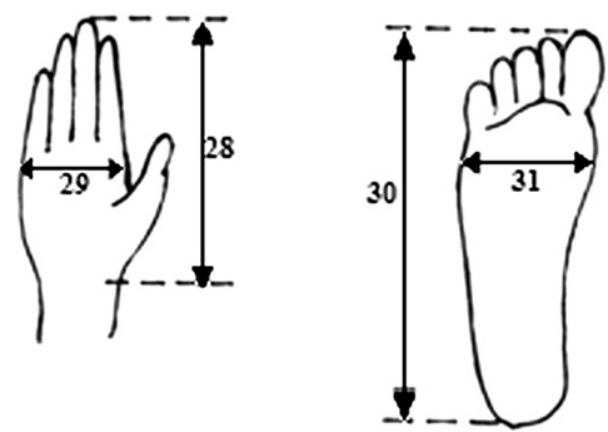

Fig. 3. Body measurement in standing/sitting position. 


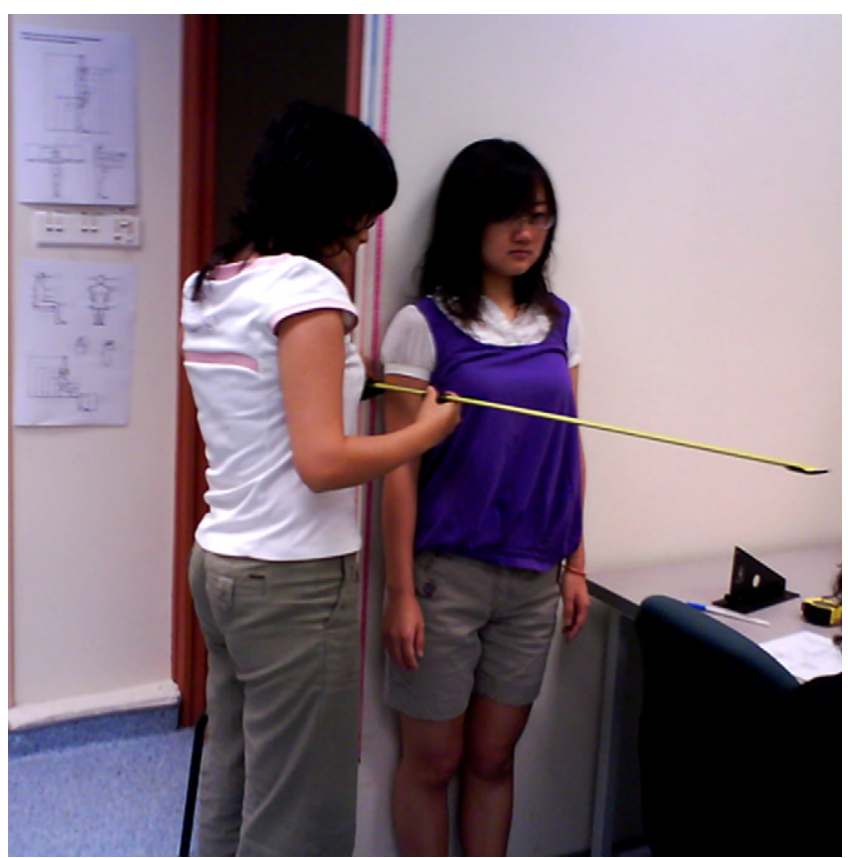

Fig. 4. A female experimenter measures a female subject. reliable and accurate as those obtained by high-tech anthropometric tools (Al-Ansari and Mokdad, 2009). For the data collection in Singapore, three sets of the Rosscraft Anthropometer Centurion Kit were used. Each set consists of 1 Campbell caliper 20, 1 Campbell caliper 10, 1 segmometer, 1 headboard, and 1 anthropometric measuring tape. A weighing scale, meter scales, and small stools were used also. For the data collection in Indonesia, a similar portable manual anthropometer, meter scales, a weighing scale, and small tools were used. All the equipments used were calibrated against standards/rules.

\subsection{Procedure}

In managing the data collection in Indonesia, four female and four male experimenters received prior training to become familiar with the equipment, body landmarks, and measurement techniques. In addition, some pilot tests were conducted. Inherently, the experimenters had an experience to be a subject of the same measurement in the previous anthropometry experiment. At the start of each data collection session, the subjects were informed of the purpose of the study, equipment, measurement procedure, and possible application of the data to be collected. The male experimenters measured the male subjects; whereas the female experimenters measured the female subjects (see Fig. 4).

In Singapore, one female and three male experimenters collected the data. The same procedure as used in Indonesia was applied in Singapore. Accuracy and repeatability in taking the

Table 1

Anthropometric data for Singaporean males (all dimensions in $\mathrm{cm}$, body weight in $\mathrm{kg}$ ).

\begin{tabular}{|c|c|c|c|c|c|c|c|c|c|c|c|c|}
\hline \multirow[t]{2}{*}{ Dimension } & \multicolumn{4}{|l|}{ Overall } & \multicolumn{4}{|l|}{ Citizens } & \multicolumn{4}{|c|}{ Chinese only } \\
\hline & 5 th & 50th & 95th & SD & 5 th & 50th & 95th & SD & 5 th & 50th & 95th & SD \\
\hline 1. Stature & 165 & 174 & 183 & 5.75 & 166 & 174 & 182 & 5.17 & 165 & 173 & 181 & 5.45 \\
\hline 2. Eye height & 153 & 163 & 172 & 6.67 & 153 & 163 & 169 & 5.03 & 153 & 162 & 169 & 5.25 \\
\hline 3. Shoulder height & 136 & 145 & 153 & 5.36 & 136 & 144 & 151 & 4.78 & 136 & 143 & 151 & 4.88 \\
\hline 4. Elbow height & 103 & 110 & 116 & 4.05 & 104 & 110 & 114 & 3.55 & 103 & 109 & 114 & 3.54 \\
\hline 5. Hip height & 86 & 97 & 106 & 5.42 & 85 & 97 & 104 & 5.32 & 85 & 96 & 104 & 5.57 \\
\hline 6. Knuckel height & 69 & 75 & 81 & 3.47 & 70 & 75 & 81 & 3.24 & 70 & 75 & 81 & 3.37 \\
\hline 7. Fingertip height & 61 & 65 & 71 & 3 & 61 & 65 & 68 & 2.69 & 61 & 65 & 68 & 2.82 \\
\hline 8. Sitting height & 84 & 90 & 96 & 4.38 & 84 & 90 & 96 & 3.43 & 85 & 90 & 97 & 3.37 \\
\hline 9. Sitting eye height & 73 & 78 & 83 & 4.9 & 73 & 77 & 83 & 4.24 & 73 & 78 & 83 & 4.53 \\
\hline 10. Sitting shoulder height & 55 & 61 & 67 & 4.49 & 55 & 61 & 66 & 3.34 & 55 & 61 & 67 & 3.42 \\
\hline 11. Sitting elbow height & 18 & 24 & 30 & 5.43 & 17 & 24 & 30 & 6.12 & 17 & 25 & 30 & 6.82 \\
\hline 12. Thigh thickness & 12 & 15 & 19 & 2.54 & 12 & 15 & 19 & 1.79 & 12 & 15 & 18 & 1.8 \\
\hline 13. Buttock-knee length & 53 & 57 & 63 & 3.34 & 53 & 57 & 63 & 2.85 & 53 & 57 & 61 & 2.52 \\
\hline 14. Buttock-popliteal length & 41 & 46 & 51 & 3.36 & 41 & 46 & 51 & 3.29 & 40 & 46 & 51 & 3.54 \\
\hline 15. Knee height & 49 & 54 & 58 & 2.89 & 50 & 54 & 58 & 2.93 & 50 & 54 & 57 & 3.05 \\
\hline 16. Popliteal height & 39 & 43 & 46 & 2.94 & 39 & 43 & 46 & 3.18 & 39 & 43 & 46 & 3 \\
\hline 17. Shoulder breadth (bideltoid) & 41 & 45 & 48 & 2.54 & 41 & 45 & 48 & 1.97 & 41 & 44 & 48 & 2.1 \\
\hline 18. Shoulder breadth (biacromial) & 31 & 37 & 43 & 3.59 & 31 & 37 & 42 & 3.5 & 31 & 37 & 41 & 3.26 \\
\hline 19. Hip breadth & 32 & 35 & 39 & 2.33 & 32 & 35 & 38 & 2 & 32 & 35 & 38 & 1.89 \\
\hline 20. Chest (bust) depth & 18 & 21 & 25 & 2.46 & 18 & 21 & 25 & 2.19 & 18 & 20 & 25 & 2.21 \\
\hline 21. Abdominal depth & 19 & 22 & 27 & 2.76 & 19 & 22 & 27 & 2.65 & 19 & 22 & 27 & 2.78 \\
\hline 22. Shoulder-elbow length & 33 & 37 & 43 & 5.99 & 33 & 37 & 43 & 7 & 32 & 37 & 43 & 7.85 \\
\hline 23. Elbow-fingertip length & 43 & 47 & 51 & 3.22 & 43 & 47 & 51 & 2.41 & 43 & 47 & 50 & 2.34 \\
\hline 24. Upper limb length & 70 & 76 & 82 & 3.55 & 71 & 76 & 81 & 3.17 & 71 & 76 & 80 & 3.01 \\
\hline 25. Shoulder-grip length & 60 & 66 & 72 & 3.37 & 61 & 66 & 71 & 3.01 & 61 & 66 & 71 & 2.95 \\
\hline 26. Head length & 18 & 19 & 21 & 1.08 & 18 & 19 & 21 & 1.03 & 18 & 19 & 21 & 1.08 \\
\hline 27. Head breadth & 15 & 17 & 19 & 1.03 & 15 & 17 & 18 & 0.99 & 15 & 17 & 18 & 1.02 \\
\hline 28. Hand length & 16 & 19 & 24 & 2.88 & 15 & 19 & 26 & 3.34 & 17 & 19 & 24 & 2.73 \\
\hline 29. Hand breadth & 8 & 9 & 10 & 0.67 & 8 & 9 & 10 & 0.58 & 8 & 9 & 10 & 0.57 \\
\hline 30. Foot length & 23 & 26 & 28 & 1.39 & 23 & 26 & 28 & 1.4 & 22 & 26 & 28 & 1.51 \\
\hline 31. Foot breadth & 9 & 10 & 11 & 0.55 & 9 & 10 & 11 & 0.53 & 9 & 10 & 11 & 0.57 \\
\hline 32. Span & 162 & 175 & 186 & 7.43 & 165 & 175 & 185 & 6.6 & 164 & 174 & 183 & 6.36 \\
\hline 33. Elbow span & 82 & 91 & 98 & 5.91 & 82 & 91 & 98 & 6.29 & 82 & 91 & 98 & 6.81 \\
\hline 34. Vertical grip reach (standing) & 194 & 208 & 224 & 15.28 & 196 & 208 & 223 & 15.1 & 195 & 207 & 221 & 16.46 \\
\hline 35. Vertical grip reach (sitting) & 117 & 124 & 132 & 5.39 & 117 & 124 & 131 & 4.39 & 117 & 123 & 131 & 4.5 \\
\hline 36. Forward grip reach & 70 & 75 & 80 & 3.25 & 70 & 75 & 80 & 3.16 & 70 & 74 & 79 & 3.21 \\
\hline 37. Body weight (kg) & 55.00 & 68.50 & 80.32 & 7.77 & 55.00 & 68.00 & 79.00 & 6.89 & 55.00 & 67.60 & 79.00 & 7.34 \\
\hline
\end{tabular}


measurements were achieved somewhat with practice prior to data collection. Initially, it took about $30 \mathrm{~min}$ to completely measure each subject. Very quickly, this plateaued to about 15-20 min.

\section{Results}

\subsection{Anthropometric data of the Singaporean and Indonesian samples}

A summary of the anthropometry data collected in Singapore is shown in Table 1 (for male) and Table 2 (for female). Table 3 presents the same for the Indonesia data. Information on the subgroups and average weight are shown.

\subsection{Comparison of selected median values for the Singaporean and Indonesian samples}

The Mann-Whitney $U$ test is used to assess whether the two independent samples (i.e., anthropometric data of Singaporean and Indonesian samples) are different. It assumes distribution-free data which is a reasonable assumption to be made of the anthropometry data collected in this study.

Table 4 presents a comparison of the 36 dimensions measured, including weight. In general, both male and female Singaporeans tend to have larger dimensions than their Indonesians counterparts. Statistical analysis shows that up to 50 percent of the dimensions have significant differences in median values. For the sub-group of citizens, 23 out of 36 for male and 21 out of 36 for female, were significantly different. For the sub-group of Chinese, 10 out of 36 for male and 15 out of 36 for female, were significantly different.

Comparing Singaporean to Indonesian male and female in the citizen sub-group and ethnic Chinese sub-group, there were significant differences in the dimensions of, stature, eye height, elbow height, fingertip height, head length, and elbow span.

\subsection{Comparison of current and past anthropometric data of Singaporeans}

The current Singaporean anthropometric data were compared with older similar data obtained in 1990. From the current data, only information from the citizen sub-group was used. The past data are from Lim et al. (1990). The sample size included in the study by Lim et al. (1990) was 587 male and 464 female Singaporean workers. The ages of the samples ranged from 18 to 45 years. The two data sets do not correspond completely. Twentyfour body dimensions were selected for comparison. Using the Student's $t$-test for independent mean difference, the result showed that more than 50 percent of the dimensions were significantly different, including weight, stature, eye height, knuckle height, sitting height, sitting eye height, sitting shoulder height, and others (see Table 5).

Table 2

Anthropometric data for Singaporean females (all dimensions in $\mathrm{cm}$, body weight in $\mathrm{kg}$ )

\begin{tabular}{|c|c|c|c|c|c|c|c|c|c|c|c|c|}
\hline \multirow[t]{2}{*}{ Dimension } & \multicolumn{4}{|l|}{ Overall } & \multicolumn{4}{|l|}{ Citizens } & \multicolumn{4}{|c|}{ Chinese } \\
\hline & 5 th & 50th & 95th & SD & 5 th & 50th & 95th & SD & 5 th & 50th & 95th & $\mathrm{SD}$ \\
\hline 1. Stature & 154 & 162 & 168 & 4.23 & 154 & 162 & 166 & 3.66 & 155 & 162 & 166 & 3.69 \\
\hline 2. Eye height & 143 & 150 & 156 & 5.41 & 143 & 150 & 155 & 3.66 & 143 & 150 & 155 & 3.61 \\
\hline 3. Shoulder height & 127 & 134 & 139 & 3.83 & 128 & 134 & 139 & 3.35 & 127 & 133 & 139 & 3.47 \\
\hline 4. Elbow height & 98 & 102 & 108 & 3.5 & 98 & 103 & 106 & 3.55 & 98 & 102 & 107 & 3.77 \\
\hline 5. Hip height & 85 & 89 & 95 & 3.26 & 86 & 89 & 94 & 2.38 & 86 & 89 & 94 & 2.39 \\
\hline 6. Knuckel height & 67 & 71 & 76 & 2.49 & 67 & 71 & 73 & 1.88 & 67 & 71 & 73 & 1.92 \\
\hline 7. Fingertip height & 58 & 62 & 67 & 2.57 & 59 & 62 & 65 & 2.11 & 58 & 62 & 65 & 2.14 \\
\hline 8. Sitting height & 81 & 85 & 91 & 3.41 & 81 & 85 & 89 & 3.25 & 81 & 85 & 89 & 3.47 \\
\hline 9. Sitting eye height & 70 & 74 & 80 & 3.5 & 70 & 74 & 78 & 3.42 & 71 & 74 & 79 & 3.67 \\
\hline 10. Sitting shoulder height & 53 & 58 & 62 & 2.5 & 55 & 58 & 61 & 2.02 & 55 & 57 & 61 & 2.12 \\
\hline 11. Sitting elbow height & 19 & 25 & 31 & 3.17 & 22 & 25 & 29 & 2.31 & 21 & 25 & 29 & 2.44 \\
\hline 12. Thigh thickness & 12 & 14 & 16 & 1.33 & 13 & 14 & 16 & 1.15 & 13 & 14 & 16 & 1.23 \\
\hline 13. Buttock-knee length & 51 & 54 & 58 & 2.72 & 51 & 54 & 58 & 1.87 & 51 & 54 & 58 & 1.91 \\
\hline 14. Buttock-popliteal length & 42 & 45 & 49 & 2.19 & 43 & 45 & 48 & 1.84 & 43 & 45 & 49 & 1.94 \\
\hline 15. Knee height & 46 & 49 & 51 & 2.18 & 46 & 49 & 51 & 1.81 & 46 & 49 & 51 & 1.91 \\
\hline 16. Popliteal height & 38 & 40 & 42 & 1.32 & 38 & 40 & 42 & 1.28 & 38 & 40 & 42 & 1.35 \\
\hline 17. Shoulder breadth (bideltoid) & 37 & 40 & 43 & 2.1 & 38 & 40 & 43 & 1.85 & 38 & 40 & 43 & 1.97 \\
\hline 18. Shoulder breadth (biacromial) & 31 & 34 & 38 & 2.58 & 31 & 34 & 36 & 1.95 & 31 & 34 & 36 & 2.03 \\
\hline 19. Hip breadth & 32 & 35 & 39 & 2.32 & 32 & 35 & 37 & 2.42 & 32 & 35 & 38 & 2.59 \\
\hline 20. Chest (bust) depth & 20 & 23 & 27 & 2.84 & 20 & 23 & 25 & 1.94 & 20 & 23 & 25 & 2.07 \\
\hline 21. Abdominal depth & 17 & 20 & 23 & 2.88 & 17 & 20 & 22 & 1.77 & 16 & 20 & 22 & 1.77 \\
\hline 22. Shoulder-elbow length & 30 & 33 & 36 & 2.39 & 30 & 33 & 35 & 1.69 & 30 & 33 & 35 & 1.75 \\
\hline 23. Elbow-fingertip length & 40 & 43 & 45 & 1.52 & 40 & 43 & 44 & 1.11 & 41 & 42 & 44 & 1.13 \\
\hline 24. Upper limb length & 66 & 70 & 73 & 2.35 & 66 & 69 & 72 & 1.75 & 67 & 69 & 72 & 1.76 \\
\hline 25. Shoulder-grip length & 57 & 60 & 65 & 3 & 57 & 60 & 62 & 1.76 & 57 & 60 & 62 & 1.74 \\
\hline 26. Head length & 17 & 18 & 19 & 0.74 & 17 & 18 & 19 & 0.46 & 17 & 18 & 19 & 0.45 \\
\hline 27. Head breadth & 15 & 16 & 17 & 0.66 & 15 & 16 & 17 & 0.5 & 15 & 16 & 17 & 0.52 \\
\hline 28. Hand length & 16 & 17 & 18 & 0.68 & 16 & 17 & 18 & 0.55 & 16 & 17 & 18 & 0.57 \\
\hline 29. Hand breadth & 7 & 7 & 8 & 0.62 & 7 & 7 & 8 & 0.62 & 7 & 7 & 8 & 0.67 \\
\hline 30. Foot length & 22 & 23 & 25 & 0.89 & 22 & 23 & 24 & 0.8 & 22 & 23 & 25 & 0.85 \\
\hline 31. Foot breadth & 8 & 9 & 10 & 0.52 & 8 & 9 & 9 & 0.37 & 8 & 9 & 9 & 0.39 \\
\hline 32. Span & 149 & 158 & 169 & 10.02 & 149 & 157 & 166 & 5.43 & 151 & 157 & 166 & 5.28 \\
\hline 33. Elbow span & 79 & 84 & 91 & 7.87 & 78 & 84 & 91 & 3.89 & 78 & 84 & 90 & 3.76 \\
\hline 34. Vertical grip reach (standing) & 179 & 189 & 202 & 14.07 & 179 & 188 & 197 & 8 & 179 & 187 & 197 & 8.39 \\
\hline 35. Vertical grip reach (sitting) & 109 & 116 & 126 & 5.45 & 110 & 115 & 121 & 4.04 & 110 & 115 & 121 & 4.2 \\
\hline 36. Forward grip reach & 63 & 68 & 73 & 2.88 & 64 & 67 & 70 & 1.98 & 64 & 67 & 70 & 2.02 \\
\hline 37. Body weight $(\mathrm{kg})$ & 46.40 & 53 & 62 & 5.42 & 46.20 & 55 & 69.70 & 6.73 & 47.60 & 52.90 & 61.79 & 4.94 \\
\hline
\end{tabular}


Table 3

Anthropometric data for Indonesian males and females (all dimensions in $\mathrm{cm}$, body weight in $\mathrm{kg}$ ).

\begin{tabular}{|c|c|c|c|c|c|c|c|c|c|c|c|c|c|c|c|c|}
\hline \multirow[t]{2}{*}{ Dimension } & \multicolumn{4}{|c|}{ Male citizens } & \multicolumn{4}{|c|}{ Male Chinese } & \multicolumn{4}{|c|}{ Female citizens } & \multicolumn{4}{|c|}{ Female Chinese } \\
\hline & 5 th & 50th & 95th & SD & 5 th & 50 th & 95th & SD & 5 th & 50th & 95th & SD & 5 th & 50th & 95th & SD \\
\hline 1. Stature & 162 & 172 & 183 & 6.23 & 165 & 171 & 180 & 4.81 & 150 & 159 & 169 & 5.76 & 151 & 159 & 166 & 5.06 \\
\hline 2. Eye height & 151 & 160 & 172 & 6.3 & 153 & 160 & 169 & 5.08 & 139 & 148 & 158 & 6.12 & 137 & 146 & 158 & 6.73 \\
\hline 3. Shoulder height & 134 & 143 & 155 & 6.41 & 134 & 143 & 151 & 5.05 & 123 & 132 & 141 & 5.91 & 123 & 132 & 139 & 5.43 \\
\hline 4. Elbow height & 99 & 107 & 114 & 5.12 & 99 & 106 & 112 & 4.29 & 91 & 99 & 108 & 6.4 & 92 & 98 & 107 & 5.35 \\
\hline 5. Hip height & 83 & 95 & 105 & 6.76 & 81 & 94 & 103 & 6.48 & 78 & 88 & 97 & 5.91 & 79 & 90 & 96 & 5.68 \\
\hline 6. Knuckel height & 68 & 75 & 82 & 4.75 & 69 & 74 & 80 & 5.13 & 63 & 70 & 78 & 4.37 & 64 & 69 & 77 & 3.89 \\
\hline 7. Fingertip height & 58 & 64 & 71 & 4.82 & 59 & 64 & 70 & 5.13 & 54 & 60 & 65 & 3.67 & 53 & 60 & 68 & 3.99 \\
\hline 8. Sitting height & 80 & 89 & 96 & 5.24 & 85 & 90 & 96 & 6.55 & 78 & 83 & 90 & 4.7 & 79 & 84 & 88 & 2.97 \\
\hline 9. Sitting eye height & 69 & 76 & 84 & 4.58 & 72 & 78 & 85 & 6.54 & 67 & 73 & 80 & 5.83 & 68 & 72 & 79 & 3.64 \\
\hline 10. Sitting shoulder height & 52 & 59 & 67 & 6.27 & 55 & 61 & 72 & 7.15 & 51 & 56 & 63 & 4.94 & 52 & 57 & 64 & 3.67 \\
\hline 11. Sitting elbow height & 19 & 24 & 30 & 4.74 & 19 & 25 & 31 & 7.13 & 19 & 25 & 32 & 5.19 & 21 & 24 & 30 & 3.24 \\
\hline 12. Thigh thickness & 12 & 16 & 22 & 3.59 & 13 & 16 & 20 & 2.76 & 11 & 15 & 19 & 3.22 & 12 & 15 & 19 & 2.81 \\
\hline 13. Buttock-knee length & 48 & 56 & 64 & 4.89 & 49 & 57 & 64 & 4.83 & 45 & 53 & 60 & 4.81 & 48 & 53 & 60 & 4.06 \\
\hline 14. Buttock-popliteal length & 40 & 46 & 54 & 4.82 & 38 & 47 & 56 & 5.36 & 37 & 43 & 51 & 4.21 & 39 & 44 & 52 & 3.97 \\
\hline 15. Knee height & 46 & 54 & 62 & 5.21 & 44 & 53 & 61 & 5.65 & 43 & 50 & 60 & 5.27 & 42 & 49 & 60 & 5.38 \\
\hline 16. Popliteal height & 38 & 44 & 49 & 3.78 & 36 & 44 & 50 & 5.36 & 38 & 44 & 50 & 3.92 & 36 & 43 & 47 & 3.85 \\
\hline 17. Shoulder breadth (bideltoid) & 36 & 45 & 52 & 4.66 & 38 & 45 & 50 & 4.6 & 37 & 43 & 53 & 5.43 & 40 & 44 & 53 & 4.97 \\
\hline 18. Shoulder breadth (biacromial) & 31 & 37 & 43 & 3.61 & 33 & 38 & 44 & 3.83 & 33 & 38 & 44 & 3.56 & 34 & 38 & 44 & 3.18 \\
\hline 19. Hip breadth & 28 & 35 & 43 & 4.41 & 30 & 35 & 44 & 4.09 & 29 & 35 & 45 & 7.22 & 30 & 34 & 42 & 4.21 \\
\hline 20. Chest (bust) depth & 16 & 21 & 27 & 3.5 & 17 & 22 & 27 & 4.02 & 17 & 21 & 28 & 3.38 & 19 & 23 & 28 & 3.61 \\
\hline 21. Abdominal depth & 15 & 21 & 29 & 4.46 & 15 & 21 & 30 & 5.19 & 14 & 18 & 25 & 3.44 & 15 & 20 & 26 & 3.93 \\
\hline 22. Shoulder-elbow length & NA & NA & NA & NA & NA & NA & NA & NA & NA & NA & NA & NA & NA & NA & NA & NA \\
\hline 23. Elbow-fingertip length & 42 & 47 & 56 & 4.55 & 41 & 46 & 53 & 4.27 & 37 & 43 & 50 & 4.27 & 37 & 42 & 47 & 3.72 \\
\hline 24. Upper limb length & 68 & 76 & 84 & 6.39 & 68 & 75 & 85 & 5.06 & 62 & 70 & 77 & 4.69 & 64 & 68 & 74 & 3.92 \\
\hline 25. Shoulder-grip length & 56 & 65 & 73 & 6.29 & 59 & 66 & 74 & 5.13 & 54 & 60 & 68 & 4.3 & 54 & 60 & 68 & 4.64 \\
\hline 26. Head length & 17 & 20 & 24 & 2.21 & 17 & 20 & 24 & 2.58 & 15 & 18 & 22 & 3.95 & 15 & 19 & 22 & 2.13 \\
\hline 27. Head breadth & 15 & 18 & 22 & 2.06 & 15 & 18 & 21 & 1.89 & 14 & 17 & 21 & 2.48 & 14 & 18 & 21 & 2.11 \\
\hline 28. Hand length & 17 & 19 & 22 & 1.64 & 15 & 19 & 22 & 2.42 & 16 & 18 & 20 & 1.72 & 17 & 18 & 20 & 2.16 \\
\hline 29. Hand breadth & 7 & 9 & 11 & 1.09 & 8 & 9 & 11 & 0.89 & 6 & 8 & 10 & 4.85 & 6 & 8 & 9 & 0.73 \\
\hline 30. Foot length & 22 & 25 & 29 & 2.58 & 11 & 25 & 28 & 4.43 & 21 & 23 & 26 & 2.63 & 21 & 23 & 26 & 2.3 \\
\hline 31. Foot breadth & 8 & 10 & 12 & 3.96 & 8 & 10 & 12 & 1.16 & 7 & 9 & 11 & 2.2 & 7 & 9 & 10 & 1.08 \\
\hline 32. Span & 158 & 172 & 186 & 8.5 & 155 & 171 & 182 & 8.73 & 146 & 156 & 170 & 7.61 & 150 & 159 & 168 & 6.52 \\
\hline 33. Elbow span & 78 & 86 & 96 & 5.97 & 79 & 87 & 94 & 4.36 & 73 & 79 & 89 & 5.38 & 73 & 81 & 88 & 4.53 \\
\hline 34. Vertical grip reach (standing) & 192 & 206 & 221 & 10.54 & 197 & 206 & 222 & 7.74 & 174 & 186 & 204 & 9.1 & 176 & 189 & 202 & 8.07 \\
\hline 35. Vertical grip reach (sitting) & 112 & 122 & 136 & 7.9 & 116 & 123 & 130 & 5.18 & 101 & 113 & 124 & 7.2 & 106 & 115 & 128 & 10.25 \\
\hline 36. Forward grip reach & 64 & 73 & 81 & 5.89 & 66 & 74 & 81 & 4.7 & 61 & 67 & 76 & 4.39 & 60 & 67 & 74 & 4.76 \\
\hline 37. Body weight (kg) & 50 & 63 & 89.25 & 13.19 & 53.05 & 63 & 93.45 & 13.35 & 39.80 & 53 & 80 & 11.68 & 41.90 & 55 & 70.40 & 9.49 \\
\hline
\end{tabular}

\subsection{Body Mass Index (BMI) as a utilization of anthropometric data}

BMI is a comparison of a person's weight against stature. It is defined as weight in $\mathrm{kg}$ divided by the square of stature in $\mathrm{m}(\mathrm{kg} /$ $\mathrm{m}^{2}$ ). Essentially, BMI can be a health indicator. The BMI values for all sub-groups in this research are shown in Table 6. All values are within the normal range of 18.5-25.0, as specified by WHO (2008). The presented BMI in this study is only valid to explain the health condition of the Singaporean and Indonesian populations in their productive age since the samples were taken from people in the age range of $18-45$ years.

\subsection{Empirical estimation of unknown anthropometric characteristics using the Ratio Scaling Method}

Pheasant and Haslegrave (2006) proposed that if given that two populations A and B which are similar in terms of age range, gender, and ethnicity, and if the parameters of variables $x$ and $y$ are known in population $A$ (i.e., the reference population), but only the parameters of $x$ are known in population B (i.e., the target population), then:

$m_{y} / m_{x}($ inreferencepopulation $\mathrm{A}) \approx m_{y} / m_{x}($ intargetpopulationB $)$

$s_{y} / s_{x}$ (in reference population $\left.\mathrm{A}\right) \approx s_{y} / s_{x}$ (in target population $\mathrm{B}$ )
The variable $x$, which is known in both populations, is called as the "scaling dimension." Stature or body height is commonly chosen as the scaling dimension and will be used here. Pheasant and Haslegrave proposed the simplest technique of coefficients calculation as follows:

$E_{1}=($ mean of required dimension $) /($ mean stature $)$

$E_{2}=$ (standard deviation of required dimension)/ (standard deviation of stature)

The $E_{1}$ and $E_{2}$ coefficients calculated from the Singaporean and Indonesian anthropometric data for two sub-groups (i.e., citizen subgroup, ethnic Chinese sub-group) are presented in Tables 7 and 8.

The objective of this exercise is to show that it is possible to estimate various anthropometric dimensions of a sample (e.g., mean, SD, percentiles) based only on the dimension of stature. However as mentioned, one requirement is that the ethnicity, age, and gender group must be the same. In further method development, Wang and Chao (2010) have developed an improvement of this Ratio Scaling Method. They called it the Constant Body Ratio benchmarks (CBR benchmarks) which is least affected by age and gender factors. Pheasant (1982) argued that when properly qualified in use, the error estimate of this technique can be considered acceptable.

A comparison of the $E_{1}$ coefficient between the current and previous studies of Singaporean citizen anthropometry is presented (see Table 9). The ratio $E_{1}$ difference (shown in “\%diff”) 
Table 4

Comparison of median values for Singaporean and Indonesian samples (all dimensions in $\mathrm{cm}$, body weight in $\mathrm{kg}$ ).

\begin{tabular}{|c|c|c|c|c|c|c|c|c|c|c|c|c|}
\hline \multirow[t]{2}{*}{ Dimension } & \multicolumn{3}{|c|}{ Male citizens } & \multicolumn{3}{|c|}{ Male Chinese } & \multicolumn{3}{|c|}{ Female citizens } & \multicolumn{3}{|c|}{ Female Chinese } \\
\hline & A & B & $p$-value & A & B & $p$-value & A & B & $p$-value & A & B & $p$-value \\
\hline 1. Stature & 172 & 174 & $0.0005^{*}$ & 171 & 173 & $0.0089^{*}$ & 159 & 162 & $0.0026^{*}$ & 159 & 162 & $0.0051^{*}$ \\
\hline 2. Eye height & 160 & 163 & $0^{*}$ & 160 & 162 & $0.0013^{*}$ & 148 & 150 & $0.0082^{*}$ & 146 & 150 & $0.0056^{*}$ \\
\hline 3. Shoulder height & 143 & 144 & $0.0371^{*}$ & 143 & 143 & 0.2343 & 132 & 134 & $0.0156^{*}$ & 132 & 133 & $0.0481^{*}$ \\
\hline 4. Elbow height & 107 & 110 & $0^{*}$ & 106 & 109 & $0^{*}$ & 99 & 103 & $0^{*}$ & 98 & 102 & $0.0005^{*}$ \\
\hline 5. Hip height & 95 & 97 & $0.0015^{*}$ & 94 & 96 & 0.0571 & 88 & 89 & $0.0016^{*}$ & 90 & 89 & 0.3176 \\
\hline 6. Knuckel height & 75 & 75 & 0.5019 & 74 & 75 & 0.1891 & 70 & 71 & 0.1613 & 69 & 71 & 0.0879 \\
\hline 7. Fingertip height & 64 & 65 & $0.0243^{*}$ & 64 & 65 & $0.019^{*}$ & 60 & 62 & $0^{*}$ & 60 & 62 & $0.0005^{*}$ \\
\hline 8. Sitting height & 89 & 90 & $0.0002^{*}$ & 90 & 90 & 0.3991 & 83 & 85 & $0.0003^{*}$ & 84 & 85 & $0.0198^{*}$ \\
\hline 9. Sitting eye height & 76 & 77 & $0^{*}$ & 78 & 78 & 0.3829 & 73 & 74 & $0.0017^{*}$ & 72 & 74 & $0.0125^{*}$ \\
\hline 10. Sitting shoulder height & 59 & 61 & $0^{*}$ & 62 & 61 & 0.6355 & 56 & 58 & $0.0052^{*}$ & 57 & 57 & 0.3485 \\
\hline 11. Sitting elbow height & 24 & 24 & 0.9111 & 25 & 25 & 0.7932 & 25 & 25 & 0.2003 & 24 & 25 & 0.0868 \\
\hline 12. Thigh thickness & 16 & 15 & $0.0007^{*}$ & 16 & 15 & $0.0075^{*}$ & 15 & 14 & 0.2182 & 15 & 14 & 0.6055 \\
\hline 13. Buttock-knee length & 56 & 57 & $0.0272^{*}$ & 57 & 57 & 0.87 & 53 & 54 & $0.001^{*}$ & 53 & 54 & 0.1889 \\
\hline 14. Buttock-popliteal length & 46 & 46 & 0.4569 & 47 & 46 & 0.1332 & 43 & 45 & $0^{*}$ & 44 & 45 & $0.0404^{*}$ \\
\hline 15. Knee height & 54 & 54 & 0.2232 & 53 & 54 & 0.1741 & 50 & 49 & $0.002^{*}$ & 49 & 49 & 0.1475 \\
\hline 16. Popliteal height & 44 & 43 & $0.0127^{*}$ & 44 & 43 & $0.0296^{*}$ & 44 & 40 & $0^{*}$ & 43 & 40 & $0.0002^{*}$ \\
\hline 17. Shoulder breadth (bideltoid) & 45 & 45 & 0.933 & 45 & 44 & 0.9665 & 43 & 40 & $0^{*}$ & 44 & 40 & $0^{*}$ \\
\hline 18. Shoulder breadth (biacromial) & 37 & 37 & 0.7922 & 38 & 37 & $0.0496^{*}$ & 38 & 34 & $0^{*}$ & 38 & 34 & $0^{*}$ \\
\hline 19. Hip breadth & 35 & 35 & 0.1687 & 35 & 35 & 0.1774 & 35 & 35 & 0.3078 & 34 & 35 & 0.9284 \\
\hline 20. Chest (bust) depth & 21 & 21 & 0.254 & 22 & 21 & 0.1353 & 21 & 23 & $0.0019^{*}$ & 23 & 23 & 0.9687 \\
\hline 21. Abdominal depth & 21 & 22 & $0^{*}$ & 21 & 22 & 0.4464 & 18 & 20 & $0.0005^{*}$ & 20 & 20 & 0.9821 \\
\hline 22. Shoulder-elbow length & NA & NA & NA & NA & NA & NA & NA & NA & NA & NA & NA & NA \\
\hline 23. Elbow-fingertip length & 47 & 47 & 0.6452 & 46 & 47 & 0.6657 & 43 & 43 & 0.7043 & 42 & 42 & 0.6493 \\
\hline 24. Upper limb length & 76 & 76 & 0.7576 & 75 & 76 & 0.9131 & 70 & 69 & 0.476 & 68 & 69 & 0.6902 \\
\hline 25. Shoulder-grip length & 65 & 66 & 0.08 & 66 & 66 & 0.5971 & 60 & 60 & 0.8928 & 60 & 60 & 0.9731 \\
\hline 26. Head length & 20 & 19 & $0.0221^{*}$ & 20 & 19 & 0.2596 & 18 & 18 & 0.1938 & 19 & 18 & 0.5251 \\
\hline 27. Head breadth & 18 & 17 & $0^{*}$ & 18 & 17 & $0^{*}$ & 17 & 16 & $0.0003^{*}$ & 18 & 16 & $0.0004^{*}$ \\
\hline 28. Hand length & 19 & 19 & 0.9333 & 19 & 19 & 0.4641 & 18 & 17 & $0.0014^{*}$ & 18 & 17 & $0^{*}$ \\
\hline 29. Hand breadth & 9 & 9 & 0.1064 & 9 & 9 & 0.2885 & 8 & 7 & 0.0522 & 8 & 7 & 0.462 \\
\hline 30. Foot length & 25 & 26 & $0.0013^{*}$ & 25 & 26 & $0.0023^{*}$ & 23 & 23 & 0.0842 & 23 & 23 & 0.0588 \\
\hline 31. Foot breadth & 10 & 10 & $0.0097^{*}$ & 10 & 10 & 0.1544 & 9 & 9 & 0.1574 & 9 & 9 & $0.0234^{*}$ \\
\hline 32. Span & 172 & 175 & $0.0009^{*}$ & 171 & 174 & 0.0726 & 156 & 157 & 0.4114 & 159 & 157 & 0.5898 \\
\hline 33. Elbow span & 86 & 91 & $0^{*}$ & 87 & 91 & $0.0002^{*}$ & 79 & 84 & $0^{*}$ & 81 & 84 & $0.0079 *$ \\
\hline 34. Vertical grip reach (standing) & 206 & 208 & $0.0058^{*}$ & 205 & 207 & 0.2913 & 186 & 188 & 0.3456 & 189 & 187 & 0.9373 \\
\hline 35. Vertical grip reach (sitting) & 122 & 124 & $0.0042^{*}$ & 122 & 123 & 0.2159 & 113 & 115 & $0.0024^{*}$ & 115 & 115 & 0.4967 \\
\hline 36. Forward grip reach & 73 & 75 & $0.0001^{*}$ & 74 & 75 & 0.312 & 67 & 67 & 0.44 & 67 & 67 & 0.6778 \\
\hline 37. Body weight (kg) & 63 & 68 & $0.0001^{*}$ & 63 & 67.6 & 0.0618 & 53 & 55 & 0.2398 & 55 & 52.9 & 0.7873 \\
\hline
\end{tabular}

*Significant at $p<0.05 ; \mathrm{A}=$ Indonesian; $\mathrm{B}=$ Singaporean.

Table 5

Comparison of 50th percentile values for previous (old) and current studies (new) of Singaporeans (all dimensions in cm, body weight in kg).

\begin{tabular}{|c|c|c|c|c|c|c|c|c|c|c|}
\hline \multirow[t]{2}{*}{ Dimension } & \multicolumn{5}{|c|}{ Male citizens } & \multicolumn{5}{|c|}{ Female citizens } \\
\hline & 50th Old & SD Old & 50th New & SD New & $p$-value & 50th Old & SD Old & 50th New & SD New & $p$-value \\
\hline 1. Stature & 169 & 6.21 & 174 & 5.17 & $0^{*}$ & 155 & 5.8 & 162 & 3.66 & $0^{*}$ \\
\hline 2. Eye height & 158 & 6.3 & 163 & 5.03 & $0^{*}$ & 144 & 5.57 & 150 & 3.66 & $0^{*}$ \\
\hline 3. Knuckel height & 78 & 5.23 & 75 & 3.24 & $0^{*}$ & 74 & 5.45 & 71 & 1.88 & $0^{*}$ \\
\hline 4. Sitting height & 87 & 4.14 & 90 & 3.43 & $0^{*}$ & 80 & 4.51 & 85 & 3.25 & $0^{*}$ \\
\hline 5. Sitting eye height & 75 & 4.36 & 77 & 4.24 & $0^{*}$ & 70 & 4.5 & 74 & 3.42 & $0^{*}$ \\
\hline 6. Sitting shoulder height & 58 & 4.74 & 61 & 3.34 & $0^{*}$ & 55 & 3.92 & 58 & 2.02 & $0^{*}$ \\
\hline 7. Sitting elbow height & 23 & 3.64 & 24 & 6.12 & $0.028^{*}$ & 23 & 3.31 & 25 & 2.31 & $0^{*}$ \\
\hline 8. Thigh thickness & 14 & 2.58 & 15 & 1.79 & $0^{*}$ & 14 & 3.9 & 14 & 1.15 & $0.012^{*}$ \\
\hline 9. Buttock-knee length & 56 & 3.44 & 57 & 2.85 & $0^{*}$ & 54 & 3.85 & 54 & 1.87 & 0.514 \\
\hline 10. Buttock-popliteal length & 45 & 3.6 & 46 & 3.29 & $0.001^{*}$ & 44 & 3.93 & 45 & 1.84 & $0^{*}$ \\
\hline 11. Knee height & 54 & 3.28 & 54 & 2.93 & 0.482 & 49 & 4.35 & 49 & 1.81 & 1 \\
\hline 12. Popliteal height & 43 & 3.36 & 43 & 3.18 & 0.512 & 40 & 4.23 & 40 & 1.28 & 0.441 \\
\hline 13. Shoulder breadth (bideltoid) & 43 & 2.75 & 45 & 1.97 & $0^{*}$ & 38 & 2.78 & 40 & 1.85 & $0^{*}$ \\
\hline 14. Hip breadth & 34 & 3.86 & 35 & 2 & $0^{*}$ & 35 & 3.64 & 35 & 2.42 & 0.582 \\
\hline 15. Chest (bust) depth & 21 & 3.02 & 21 & 2.19 & 0.076 & 22 & 2.87 & 23 & 1.94 & $0.001^{*}$ \\
\hline 16. Abdominal depth & 20 & 3.03 & 22 & 2.65 & $0^{*}$ & 20 & 4.05 & 20 & 1.77 & 1 \\
\hline 17. Elbow-fingertip length & 45 & 2.65 & 47 & 2.41 & $0^{*}$ & 41 & 2.97 & 43 & 1.11 & $0^{*}$ \\
\hline 18. Head length & 20 & 1.47 & 19 & 1.03 & $0^{*}$ & 19 & 1.59 & 18 & 0.46 & $0^{*}$ \\
\hline 19. Head breadth & 16 & 1.35 & 17 & 0.99 & $0^{*}$ & 16 & 1.78 & 16 & 0.5 & $0.005^{*}$ \\
\hline 20. Hand length & 19 & 1.02 & 19 & 3.34 & 0.084 & 17 & 1 & 17 & 0.55 & 1 \\
\hline 21. Hand breadth & 8 & 0.68 & 9 & 0.58 & $0^{*}$ & 7 & 0.61 & 7 & 0.62 & $0.001^{*}$ \\
\hline 22. Foot length & 25 & 1.33 & 26 & 1.4 & $0^{*}$ & 23 & 1.25 & 23 & 0.8 & $0^{*}$ \\
\hline 23. Foot breadth & 10 & 0.66 & 10 & 0.53 & $0^{*}$ & 9 & 0.67 & 9 & 0.37 & 0.088 \\
\hline 24. Weight (kg) & 60 & 7.83 & 68 & 6.89 & $0^{*}$ & 50 & 9.15 & 55 & 6.73 & $0^{*}$ \\
\hline
\end{tabular}


Table 6

Body Mass Index of Singaporean and Indonesian samples.

\begin{tabular}{llll}
\hline Subject & & \multicolumn{2}{l}{$\begin{array}{l}\text { Body Mass Index (BMI) } \\
\text { in } \mathrm{kg} / \mathrm{m}^{2}\end{array}$} \\
\cline { 3 - 4 } \cline { 3 - 3 } & & Male & Female \\
\hline Singaporean & Overall & 22.58 & 20.65 \\
& Citizens & 22.62 & 21.43 \\
& Chinese & 22.5 & 20.71 \\
& Citizen (for old data) & 21.01 & 20.81 \\
Indonesian & Citizens & 22.21 & 21.76 \\
& Chinese & 22.7 & 21.89 \\
\hline
\end{tabular}

indicates that the errors are relatively small in all body dimensions except for knuckle height and head length. The average difference is 2.28 percent for males and 3.14 percent for females.

\section{Discussion and conclusion}

Anthropometric data from Singaporean and Indonesian adults were collected and summarized. Based on statistical analysis and depending on the sub-group sample, up to half of the dimensions were significantly different. The Singaporean sample was relatively taller than the Indonesian sample for both male and female. Singaporeans were also significantly larger than Indonesians in several body dimensions including eye height, elbow height, fingertip height, and elbow span. However, Singaporean adults appear to have a relatively smaller head length than Indonesian adults.
The above indicates that geographical origin does have an effect on variation of anthropometry. This confirms with reports by other researchers. For example, Sirajuddin et al. (1994) found that geographic factors have an overwhelming effect on the genetic differentiation of ethnic groups in the southern part of Indian. İșeri and Arslan (2009) found geographical region to have a significant effect on stature and weight of the Turkish population. Another variable is the various ethnic sub-groups.

Singapore is a highly diverse and cosmopolitan country with the Chinese people forming the largest ethnic majority. Other subgroups include Malays, Indians, Eurasians, and Arabs. It is interesting to note that in Indonesia alone, there are over 300 distinct native ethnicities. On the issue of ethnicity affecting anthropometry, it is not a question of whether it does, but how much it does.

Better nutrition and higher social status may be an explanation for differences found between the two samples. Compared to Indonesia, Singapore has a higher status since its GDP (nominal) per capita (i.e., USD38,972) was ranked 22nd, whereas Indonesia (i.e., USD2239) was ranked 116th in year 2008 (International Monetary Fund, 2008). Higher socioeconomic status implies higher income and is associated with better education, resulting in better nutrition, better child care, and better medical and social services. İşeri and Arslan (2009) argue that over time, this leads to an increase in overall stature. Malnutrition which is prevalent in underdeveloped countries plays a major role in inhibiting the human growth process including height and weight (Wall, 1993). As empirical proof, in Europe during the last 100 years, people have become taller, the onset of puberty has commenced at a younger

Table 7

$E_{1}$ and $E_{2}$ coefficients calculated from Singaporean anthropometric data as reference populations.

\begin{tabular}{|c|c|c|c|c|c|c|c|c|c|c|c|c|c|c|c|c|}
\hline \multirow[t]{2}{*}{ Body dimension } & \multicolumn{4}{|c|}{ Male citizen } & \multicolumn{4}{|c|}{ Female citizen } & \multicolumn{4}{|c|}{ Male Chinese } & \multicolumn{4}{|c|}{ Female Chinese } \\
\hline & Mean & SD & $E_{1}$ & $E_{2}$ & Mean & SD & $E_{1}$ & $E_{2}$ & Mean & SD & $E_{1}$ & $E_{2}$ & Mean & SD & $E_{1}$ & $E_{2}$ \\
\hline 1. Stature & 174 & 5.17 & 1 & 1 & 162 & 3.7 & 1 & 1 & 174 & 5.45 & 1 & 1 & 161 & 3.69 & 1 & 1 \\
\hline 2. Eye height & 163 & 5.03 & 0.94 & 0.97 & 150 & 3.7 & 0.9 & 1 & 162 & 5.25 & 0.94 & 0.96 & 150 & 3.61 & 0.93 & 0.98 \\
\hline 3. Shoulder height & 144 & 4.78 & 0.83 & 0.93 & 133 & 3.4 & 0.8 & 0.9 & 144 & 4.88 & 0.83 & 0.9 & 133 & 3.47 & 0.83 & 0.94 \\
\hline 4. Elbow height & 110 & 3.55 & 0.63 & 0.69 & 103 & 3.6 & 0.6 & 1 & 109 & 3.54 & 0.63 & 0.65 & 103 & 3.77 & 0.64 & 1.02 \\
\hline 5. Hip height & 97 & 5.32 & 0.56 & 1.03 & 90 & 2.4 & 0.6 & 0.7 & 96 & 5.57 & 0.55 & 1.02 & 90 & 2.39 & 0.56 & 0.65 \\
\hline 6. Knuckel height & 75 & 3.24 & 0.43 & 0.63 & 71 & 1.9 & 0.4 & 0.5 & 75 & 3.37 & 0.43 & 0.62 & 71 & 1.92 & 0.44 & 0.52 \\
\hline 7. Fingertip height & 65 & 2.69 & 0.37 & 0.52 & 62 & 2.1 & 0.4 & 0.6 & 65 & 2.82 & 0.37 & 0.52 & 62 & 2.14 & 0.39 & 0.58 \\
\hline 8. Sitting height & 90 & 3.43 & 0.52 & 0.66 & 85 & 3.3 & 0.5 & 0.9 & 90 & 3.37 & 0.52 & 0.62 & 85 & 3.47 & 0.53 & 0.94 \\
\hline 9. Sitting eye height & 77 & 4.24 & 0.45 & 0.82 & 74 & 3.4 & 0.5 & 0.9 & 77 & 4.53 & 0.45 & 0.83 & 74 & 3.67 & 0.46 & 0.99 \\
\hline 10. Sitting shoulder height & 61 & 3.34 & 0.35 & 0.65 & 58 & 2 & 0.4 & 0.6 & 61 & 3.42 & 0.35 & 0.63 & 58 & 2.12 & 0.36 & 0.57 \\
\hline 11. Sitting elbow height & 25 & 6.12 & 0.14 & 1.18 & 25 & 2.3 & 0.2 & 0.6 & 25 & 6.82 & 0.15 & 1.25 & 25 & 2.44 & 0.16 & 0.66 \\
\hline 12. Thigh thickness & 15 & 1.79 & 0.09 & 0.35 & 14 & 1.2 & 0.1 & 0.3 & 15 & 1.8 & 0.09 & 0.33 & 14 & 1.23 & 0.09 & 0.33 \\
\hline 13. Buttock-knee length & 57 & 2.85 & 0.33 & 0.55 & 54 & 1.9 & 0.3 & 0.5 & 57 & 2.52 & 0.33 & 0.46 & 54 & 1.91 & 0.34 & 0.52 \\
\hline 14. Buttock-popliteal length & 46 & 3.29 & 0.27 & 0.64 & 45 & 1.8 & 0.3 & 0.5 & 46 & 3.54 & 0.27 & 0.65 & 46 & 1.94 & 0.28 & 0.53 \\
\hline 15. Knee height & 54 & 2.93 & 0.31 & 0.57 & 49 & 1.8 & 0.3 & 0.5 & 54 & 3.05 & 0.31 & 0.56 & 49 & 1.91 & 0.3 & 0.52 \\
\hline 16. Popliteal height & 43 & 3.18 & 0.25 & 0.62 & 40 & 1.3 & 0.2 & 0.3 & 43 & 3 & 0.25 & 0.55 & 40 & 1.35 & 0.25 & 0.37 \\
\hline 17. Shoulder breadth (bideltoid) & 44 & 1.97 & 0.26 & 0.38 & 40 & 1.9 & 0.3 & 0.5 & 44 & 2.1 & 0.25 & 0.39 & 40 & 1.97 & 0.25 & 0.53 \\
\hline 18. Shoulder breadth (biacromial) & 37 & 3.5 & 0.21 & 0.68 & 34 & 2 & 0.2 & 0.5 & 37 & 3.26 & 0.21 & 0.6 & 34 & 2.03 & 0.21 & 0.55 \\
\hline 19. Hip breadth & 35 & 2 & 0.2 & 0.39 & 35 & 2.4 & 0.2 & 0.7 & 35 & 1.89 & 0.2 & 0.35 & 35 & 2.59 & 0.22 & 0.7 \\
\hline 20. Chest (bust) depth & 21 & 2.19 & 0.12 & 0.42 & 23 & 1.9 & 0.1 & 0.5 & 21 & 2.21 & 0.12 & 0.41 & 23 & 2.07 & 0.14 & 0.56 \\
\hline 21. Abdominal depth & 22 & 2.65 & 0.13 & 0.51 & 20 & 1.8 & 0.1 & 0.5 & 22 & 2.78 & 0.13 & 0.51 & 20 & 1.77 & 0.12 & 0.48 \\
\hline 22. Shoulder-elbow length & 38 & 7 & 0.22 & 1.35 & 33 & 1.7 & 0.2 & 0.5 & 38 & 7.85 & 0.22 & 1.44 & 33 & 1.75 & 0.2 & 0.47 \\
\hline 23. Elbow-fingertip length & 47 & 2.41 & 0.27 & 0.47 & 42 & 1.1 & 0.3 & 0.3 & 47 & 2.34 & 0.27 & 0.43 & 42 & 1.13 & 0.26 & 0.31 \\
\hline 24. Upper limb length & 76 & 3.17 & 0.44 & 0.61 & 69 & 1.8 & 0.4 & 0.5 & 75 & 3.01 & 0.44 & 0.55 & 69 & 1.76 & 0.43 & 0.48 \\
\hline 25. Shoulder-grip length & 66 & 3.01 & 0.38 & 0.58 & 60 & 1.8 & 0.4 & 0.5 & 66 & 2.95 & 0.38 & 0.54 & 60 & 1.74 & 0.37 & 0.47 \\
\hline 26. Head length & 19 & 1.03 & 0.11 & 0.2 & 18 & 0.5 & 0.1 & 0.1 & 19 & 1.08 & 0.11 & 0.2 & 18 & 0.45 & 0.11 & 0.12 \\
\hline 27. Head breadth & 17 & 0.99 & 0.1 & 0.19 & 16 & 0.5 & 0.1 & 0.1 & 17 & 1.02 & 0.1 & 0.19 & 16 & 0.52 & 0.1 & 0.14 \\
\hline 28. Hand length & 19 & 3.34 & 0.11 & 0.65 & 17 & 0.6 & 0.1 & 0.1 & 19 & 2.73 & 0.11 & 0.5 & 17 & 0.57 & 0.11 & 0.15 \\
\hline 29. Hand breadth & 9 & 0.58 & 0.05 & 0.11 & 7 & 0.6 & 0 & 0.2 & 9 & 0.57 & 0.05 & 0.1 & 7 & 0.67 & 0.05 & 0.18 \\
\hline 30. Foot length & 26 & 1.4 & 0.15 & 0.27 & 23 & 0.8 & 0.1 & 0.2 & 26 & 1.51 & 0.15 & 0.28 & 23 & 0.85 & 0.14 & 0.23 \\
\hline 31. Foot breadth & 10 & 0.53 & 0.06 & 0.1 & 9 & 0.4 & 0.1 & 0.1 & 10 & 0.57 & 0.06 & 0.11 & 9 & 0.39 & 0.06 & 0.11 \\
\hline 32. Span & 175 & 6.6 & 1.01 & 1.28 & 158 & 5.4 & 1 & 1.5 & 174 & 6.36 & 1 & 1.17 & 158 & 5.28 & 0.98 & 1.43 \\
\hline 33. Elbow span & 91 & 6.29 & 0.52 & 1.22 & 84 & 3.9 & 0.5 & 1.1 & 91 & 6.81 & 0.52 & 1.25 & 84 & 3.76 & 0.52 & 1.02 \\
\hline 34. Vertical grip reach (standing) & 208 & 15.1 & 1.19 & 2.92 & 188 & 8 & 1.2 & 2.2 & 206 & 16.5 & 1.19 & 3.02 & 187 & 8.39 & 1.16 & 2.27 \\
\hline 35. Vertical grip reach (sitting) & 124 & 4.39 & 0.71 & 0.85 & 116 & 4 & 0.7 & 1.1 & 124 & 4.5 & 0.71 & 0.83 & 116 & 4.2 & 0.72 & 1.14 \\
\hline 36. Forward grip reach & 75 & 3.16 & 0.43 & 0.61 & 67 & 2 & 0.4 & 0.5 & 75 & 3.21 & 0.43 & 0.59 & 67 & 2.02 & 0.42 & 0.55 \\
\hline
\end{tabular}


Table 8

$E_{1}$ and $E_{2}$ coefficients calculated from Indonesian anthropometric data as reference populations.

\begin{tabular}{|c|c|c|c|c|c|c|c|c|c|c|c|c|c|c|c|c|}
\hline \multirow[t]{2}{*}{ Body dimension } & \multicolumn{4}{|c|}{ Male citizen } & \multicolumn{4}{|c|}{ Female citizen } & \multicolumn{4}{|c|}{ Male Chinese } & \multicolumn{4}{|c|}{ Female Chinese } \\
\hline & Mean & SD & $E_{1}$ & $E_{2}$ & Mean & SD & $E_{1}$ & $E_{2}$ & Mean & SD & $E_{1}$ & $E_{2}$ & Mean & SD & $E_{1}$ & $E_{2}$ \\
\hline 1. Stature & 172 & 6.23 & 1 & 1 & 159 & 5.8 & 1 & 1 & 171 & 4.81 & 1 & 1 & 159 & 5.06 & 1 & 1 \\
\hline 2. Eye height & 160 & 6.3 & 0.93 & 1.01 & 148 & 6.1 & 0.9 & 1.1 & 160 & 5.08 & 0.93 & 1.06 & 147 & 6.73 & 0.93 & 1.33 \\
\hline 3. Shoulder height & 143 & 6.41 & 0.83 & 1.03 & 132 & 5.9 & 0.8 & 1 & 143 & 5.05 & 0.83 & 1.05 & 132 & 5.43 & 0.83 & 1.08 \\
\hline 4. Elbow height & 107 & 5.12 & 0.62 & 0.82 & 100 & 6.4 & 0.6 & 1.1 & 106 & 4.29 & 0.62 & 0.89 & 99 & 5.35 & 0.62 & 1.06 \\
\hline 5. Hip height & 95 & 6.76 & 0.55 & 1.08 & 87 & 5.9 & 0.5 & 1 & 94 & 6.48 & 0.55 & 1.35 & 88 & 5.68 & 0.56 & 1.12 \\
\hline 6. Knuckel height & 75 & 4.75 & 0.44 & 0.76 & 70 & 4.4 & 0.4 & 0.8 & 74 & 5.13 & 0.43 & 1.07 & 70 & 3.89 & 0.44 & 0.77 \\
\hline 7. Fingertip height & 64 & 4.82 & 0.37 & 0.77 & 60 & 3.7 & 0.4 & 0.6 & 64 & 5.13 & 0.37 & 1.07 & 60 & 3.99 & 0.38 & 0.79 \\
\hline 8. Sitting height & 88 & 5.24 & 0.51 & 0.84 & 83 & 4.7 & 0.5 & 0.8 & 91 & 6.55 & 0.53 & 1.36 & 84 & 2.97 & 0.53 & 0.59 \\
\hline 9. Sitting eye height & 76 & 4.58 & 0.44 & 0.74 & 73 & 5.8 & 0.5 & 1 & 79 & 6.54 & 0.46 & 1.36 & 73 & 3.64 & 0.46 & 0.72 \\
\hline 10. Sitting shoulder height & 59 & 6.27 & 0.35 & 1.01 & 57 & 4.9 & 0.4 & 0.9 & 62 & 7.15 & 0.37 & 1.49 & 57 & 3.67 & 0.36 & 0.73 \\
\hline 11. Sitting elbow height & 25 & 4.74 & 0.14 & 0.76 & 25 & 5.2 & 0.2 & 0.9 & 26 & 7.13 & 0.15 & 1.48 & 24 & 3.24 & 0.15 & 0.64 \\
\hline 12. Thigh thickness & 16 & 3.59 & 0.1 & 0.58 & 15 & 3.2 & 0.1 & 0.6 & 17 & 2.76 & 0.1 & 0.57 & 15 & 2.81 & 0.1 & 0.56 \\
\hline 13. Buttock-knee length & 56 & 4.89 & 0.33 & 0.78 & 53 & 4.8 & 0.3 & 0.8 & 57 & 4.83 & 0.33 & 1 & 54 & 4.06 & 0.34 & 0.8 \\
\hline 14. Buttock-popliteal length & 46 & 4.82 & 0.27 & 0.77 & 43 & 4.2 & 0.3 & 0.7 & 47 & 5.36 & 0.28 & 1.11 & 45 & 3.97 & 0.28 & 0.79 \\
\hline 15. Knee height & 54 & 5.21 & 0.31 & 0.84 & 51 & 5.3 & 0.3 & 0.9 & 53 & 5.65 & 0.31 & 1.17 & 50 & 5.38 & 0.32 & 1.06 \\
\hline 16. Popliteal height & 44 & 3.78 & 0.26 & 0.61 & 44 & 3.9 & 0.3 & 0.7 & 44 & 5.36 & 0.26 & 1.11 & 43 & 3.85 & 0.27 & 0.76 \\
\hline 17. Shoulder breadth (bideltoid) & 45 & 4.66 & 0.26 & 0.75 & 45 & 5.4 & 0.3 & 0.9 & 44 & 4.6 & 0.26 & 0.96 & 45 & 4.97 & 0.28 & 0.98 \\
\hline 18. Shoulder breadth (biacromial) & 37 & 3.61 & 0.21 & 0.58 & 38 & 3.6 & 0.2 & 0.6 & 38 & 3.83 & 0.22 & 0.8 & 39 & 3.18 & 0.24 & 0.63 \\
\hline 19. Hip breadth & 36 & 4.41 & 0.21 & 0.71 & 36 & 7.2 & 0.2 & 1.3 & 36 & 4.09 & 0.21 & 0.85 & 36 & 4.21 & 0.22 & 0.83 \\
\hline 20. Chest (bust) depth & 21 & 3.5 & 0.12 & 0.56 & 21 & 3.4 & 0.1 & 0.6 & 22 & 4.02 & 0.13 & 0.84 & 23 & 3.61 & 0.14 & 0.71 \\
\hline 21. Abdominal depth & 21 & 4.46 & 0.12 & 0.72 & 19 & 3.4 & 0.1 & 0.6 & 22 & 5.19 & 0.13 & 1.08 & 20 & 3.93 & 0.13 & 0.78 \\
\hline 22. Shoulder-elbow length & NA & NA & NA & NA & NA & NA & NA & NA & NA & NA & NA & NA & NA & NA & NA & NA \\
\hline 23. Elbow-fingertip length & 48 & 4.55 & 0.28 & 0.73 & 43 & 4.3 & 0.3 & 0.7 & 47 & 4.27 & 0.27 & 0.89 & 42 & 3.72 & 0.26 & 0.74 \\
\hline 24. Upper limb length & 75 & 6.39 & 0.44 & 1.03 & 70 & 4.7 & 0.4 & 0.8 & 76 & 5.06 & 0.44 & 1.05 & 69 & 3.92 & 0.44 & 0.77 \\
\hline 25. Shoulder-grip length & 65 & 6.29 & 0.38 & 1.01 & 60 & 4.3 & 0.4 & 0.7 & 66 & 5.13 & 0.38 & 1.07 & 60 & 4.64 & 0.38 & 0.92 \\
\hline 26. Head length & 20 & 2.21 & 0.12 & 0.35 & 19 & 4 & 0.1 & 0.7 & 20 & 2.58 & 0.12 & 0.54 & 18 & 2.13 & 0.11 & 0.42 \\
\hline 27. Head breadth & 19 & 2.06 & 0.11 & 0.33 & 17 & 2.5 & 0.1 & 0.4 & 18 & 1.89 & 0.11 & 0.39 & 17 & 2.11 & 0.11 & 0.42 \\
\hline 28. Hand length & 19 & 1.64 & 0.11 & 0.26 & 18 & 1.7 & 0.1 & 0.3 & 19 & 2.42 & 0.11 & 0.5 & 18 & 2.16 & 0.11 & 0.43 \\
\hline 29. Hand breadth & 9 & 1.09 & 0.05 & 0.18 & 8 & 4.9 & 0.1 & 0.8 & 9 & 0.89 & 0.05 & 0.19 & 7 & 0.73 & 0.05 & 0.15 \\
\hline 30. Foot length & 25 & 2.58 & 0.15 & 0.41 & 23 & 2.6 & 0.1 & 0.5 & 24 & 4.43 & 0.14 & 0.92 & 23 & 2.3 & 0.15 & 0.46 \\
\hline 31. Foot breadth & 10 & 3.96 & 0.06 & 0.64 & 9 & 2.2 & 0.1 & 0.4 & 10 & 1.16 & 0.06 & 0.24 & 8 & 1.08 & 0.05 & 0.21 \\
\hline 32. Span & 172 & 8.5 & 1 & 1.36 & 157 & 7.6 & 1 & 1.3 & 172 & 8.73 & 1 & 1.81 & 158 & 6.52 & 1 & 1.29 \\
\hline 33. Elbow span & 87 & 5.97 & 0.5 & 0.96 & 79 & 5.4 & 0.5 & 0.9 & 87 & 4.36 & 0.51 & 0.91 & 81 & 4.53 & 0.51 & 0.9 \\
\hline 34. Vertical grip reach (standing) & 206 & 10.5 & 1.2 & 1.69 & 187 & 9.1 & 1.2 & 1.6 & 207 & 7.74 & 1.21 & 1.61 & 188 & 8.07 & 1.19 & 1.6 \\
\hline 35. Vertical grip reach (sitting) & 123 & 7.9 & 0.71 & 1.27 & 113 & 7.2 & 0.7 & 1.3 & 123 & 5.18 & 0.72 & 1.08 & 116 & 10.3 & 0.73 & 2.03 \\
\hline 36. Forward grip reach & 73 & 5.89 & 0.42 & 0.94 & 67 & 4.4 & 0.4 & 0.8 & 74 & 4.7 & 0.43 & 0.98 & 67 & 4.76 & 0.43 & 0.94 \\
\hline
\end{tabular}

Table 9

Comparison of $E_{1}$ and $E_{2}$ coefficients between previous (old) and current (new) studies of Singapore citizen anthropometry.

\begin{tabular}{|c|c|c|c|c|c|c|c|c|c|c|c|c|c|c|}
\hline \multirow[t]{2}{*}{ Body dimension } & \multicolumn{4}{|c|}{ Old data for male } & \multicolumn{2}{|c|}{$\begin{array}{l}\text { New data for } \\
\text { male }\end{array}$} & \multirow[t]{2}{*}{ Diff* (\%) } & \multicolumn{4}{|c|}{ Old data for female } & \multicolumn{2}{|c|}{$\begin{array}{l}\text { New data for } \\
\text { female }\end{array}$} & \multirow[t]{2}{*}{$\operatorname{Diff}^{* *}(\%)$} \\
\hline & Mean & $\mathrm{SD}$ & $E_{1}$ & $E_{2}$ & $E_{1}$ & $E_{2}$ & & Mean & SD & $E_{1}$ & $E_{2}$ & $E_{1}$ & $E_{2}$ & \\
\hline 1. Stature & 169 & 6.2 & 1 & 1 & 1 & 1 & - & 155 & 5.8 & 1 & 1 & 1 & 1 & - \\
\hline 2. Eye height & 158 & 6.3 & 0.9 & 1 & 0.936 & 0.973 & 0.08 & 144 & 5.6 & 0.9 & 1 & 0.926 & 1 & 0.38 \\
\hline 3. Knuckel height & 78 & 5.2 & 0.5 & 0.8 & 0.432 & 0.626 & 6.48 & 74 & 5.5 & 0.5 & 0.9 & 0.438 & 0.514 & 8.24 \\
\hline 4. Sitting height & 87 & 4.1 & 0.5 & 0.7 & 0.517 & 0.663 & 0.94 & 80 & 4.5 & 0.5 & 0.8 & 0.527 & 0.889 & 1.6 \\
\hline 5. Sitting eye height & 75 & 4.4 & 0.4 & 0.7 & 0.445 & 0.819 & 0.77 & 70 & 4.5 & 0.5 & 0.8 & 0.457 & 0.935 & 1.24 \\
\hline 6. Sitting shoulder height & 58 & 4.7 & 0.3 & 0.8 & 0.349 & 0.646 & 1.12 & 55 & 3.9 & 0.4 & 0.7 & 0.357 & 0.553 & 1.39 \\
\hline 7. Sitting elbow height & 23 & 3.6 & 0.1 & 0.6 & 0.139 & 1.184 & 2.19 & 23 & 3.3 & 0.1 & 0.6 & 0.154 & 0.632 & 5.47 \\
\hline 8. Thigh thickness & 14 & 2.6 & 0.1 & 0.4 & 0.087 & 0.347 & 5.45 & 14 & 3.9 & 0.1 & 0.7 & 0.088 & 0.315 & 0.01 \\
\hline 9. Buttock-knee length & 56 & 3.4 & 0.3 & 0.6 & 0.328 & 0.551 & 0.96 & 54 & 3.9 & 0.3 & 0.7 & 0.335 & 0.512 & 3.88 \\
\hline 10. Buttock-popliteal length & 45 & 3.6 & 0.3 & 0.6 & 0.265 & 0.636 & 0.5 & 44 & 3.9 & 0.3 & 0.7 & 0.279 & 0.502 & 1.62 \\
\hline 11. Knee height & 54 & 3.3 & 0.3 & 0.5 & 0.31 & 0.567 & 2.51 & 49 & 4.4 & 0.3 & 0.8 & 0.3 & 0.494 & 4.23 \\
\hline 12. Popliteal height & 43 & 3.4 & 0.3 & 0.5 & 0.248 & 0.616 & 2.42 & 40 & 4.2 & 0.3 & 0.7 & 0.248 & 0.349 & 3.75 \\
\hline 13. Shoulder breadth (bideltoid) & 43 & 2.8 & 0.3 & 0.4 & 0.256 & 0.382 & 0.63 & 38 & 2.8 & 0.2 & 0.5 & 0.248 & 0.506 & 2.66 \\
\hline 14. Hip breadth & 34 & 3.9 & 0.2 & 0.6 & 0.2 & 0.387 & 0.6 & 35 & 3.6 & 0.2 & 0.6 & 0.215 & 0.661 & 4.78 \\
\hline 15. Chest (bust) depth & 21 & 3 & 0.1 & 0.5 & 0.118 & 0.424 & 4.72 & 22 & 2.9 & 0.1 & 0.5 & 0.139 & 0.531 & 0.22 \\
\hline 16. Abdominal depth & 20 & 3 & 0.1 & 0.5 & 0.126 & 0.513 & 6.31 & 20 & 4.1 & 0.1 & 0.7 & 0.124 & 0.485 & 4.23 \\
\hline 17. Elbow-fingertip length & 45 & 2.7 & 0.3 & 0.4 & 0.268 & 0.467 & 0.69 & 41 & 3 & 0.3 & 0.5 & 0.263 & 0.303 & 0.73 \\
\hline 18. Head length & 20 & 1.5 & 0.1 & 0.2 & 0.11 & 0.199 & 6.76 & 19 & 1.6 & 0.1 & 0.3 & 0.111 & 0.126 & 10.72 \\
\hline 19. Head breadth & 16 & 1.4 & 0.1 & 0.2 & 0.095 & 0.192 & 0.14 & 16 & 1.8 & 0.1 & 0.3 & 0.099 & 0.137 & 2.4 \\
\hline 20. Hand length & 19 & 1 & 0.1 & 0.2 & 0.109 & 0.645 & 0.25 & 17 & 1 & 0.1 & 0.2 & 0.105 & 0.149 & 4.23 \\
\hline 21. Hand breadth & 8 & 0.7 & 0 & 0.1 & 0.049 & 0.112 & 3.2 & 7 & 0.6 & 0 & 0.1 & 0.046 & 0.169 & 0.19 \\
\hline 22. Foot length & 25 & 1.3 & 0.1 & 0.2 & 0.149 & 0.271 & 1.22 & 23 & 1.3 & 0.1 & 0.2 & 0.14 & 0.22 & 1.7 \\
\hline 23. Foot breadth & 10 & 0.7 & 0.1 & 0.1 & 0.058 & 0.102 & 2.19 & 9 & 0.7 & 0.1 & 0.1 & 0.055 & 0.1 & 5.3 \\
\hline Diff average & & & & & & & 2.28 & & & & & & & 3.14 \\
\hline
\end{tabular}

${ }^{*} E_{1}$ difference $=\left\{\mid\left(E_{1}\right.\right.$ old $-E_{1}$ new $) / E_{1}$ old $\left.\mid\right\} \times 100 \%$ for male; ${ }^{* *} E_{1}$ difference $=\left\{\mid\left(E_{1}\right.\right.$ old $-E_{1}$ new $) / E_{1}$ old $\left.\mid\right\} \times 100 \%$ for female. 
age, and the full-grown state has been reached at an earlier age (Wall, 1993).

In comparing the past and current anthropometric data of Singaporean adults, it is not surprising to find many dimensions significantly different (refer to Table 5 ). The results indicate that current Singapore citizens (both male and female) are larger than those in the 1990s. Some significant dimensions include stature, eye height, knuckle height, sitting height, and weight. The average stature has increased by $50 \mathrm{~mm}$ for male and $68.5 \mathrm{~mm}$ for female in Singapore over the last 19 years. More than 50 percent of the dimensions are significantly different ( 20 out of 24 for male; 17 out of 24 for female). Again, the factors of nutrition, disease, socioeconomic status, urbanization, physical activity, climate, and psychosocial deprivation, all contribute to the growth of body dimensions (Wall, 1993).

Based on the results of the BMI calculation, both Singaporeans and Indonesians for all sub-groups, have healthy body weights. Compared to the previous study of Singaporean anthropometric data, the current study indicate that the Singaporean sample (both male and female) has gained more weight, as evidenced by their higher BMI and stature.

The comparison of ratio $E_{1}$ between the existing and current Singapore citizen anthropometric data (both male and female) showed that there is relatively no difference. It is indicated by the small difference in the averages (i.e., 2.28 percent for males, 3.14 percent for females). Essentially, this finding may be utilized as a reference population to compare against another population (e.g., compare Singapore Chinese to Malays, Indians, etc). The utilization of this method was shown by İseri and Arslan (2009) who estimated Turkish adult anthropometric data. They had to assume that the body ratios of the Turkish people have not changed for the past 45 years.

In conclusion, the Singaporean sample is significantly different from the Indonesian sample in various body dimensions. In general, Singaporeans have larger dimensions than Indonesians. The current Singaporean sample is also significantly larger than past Singaporeans. Geographical origin, nutrition, social status, and ethnic composition of populations are some general factors influencing the distribution of anthropometric characteristics. Hence, a regular updating of anthropometric data is required. These could be used as relevant consideration in system design when there is a need to deal with human anthropometric variability.

Since there are significant increases in certain body dimensions with time due to changes in dietary habits, nutrition, and socioeconomic status etc as mentioned by Wall (1993), it implies that the human body dimensions (e.g. stature and body weight) can be predicted in the near future by proposing a mathematical modeling (e.g. a linear regression model of certain human body dimension which taking 'time' as an independent variable). In addition to that, it is essential to provide adjustable equipment/facilities in order to achieve comfort and proper posture. Some examples concerned with adjustability are: (i) Building a prototype of an adjustable table and chair for schools (Jung, 2005), (ii) An adjustable vice mount (Boussena and Davies, 1989), (iii) Redesigning a hospital meal cart by incorporating ergonomic principles and data (Das et al., 2002). Besides, in the future, the apparel industry can be considered as a very dramatic phenomenon due to changes in the size of the human body dimensions. For example, the current small (S), medium (M), and large (L) sizes could not be applied as a benchmark for mass production in the future; these will certainly evolve.

However, this study is far from complete. The time and resource constraints were limiting factors. Further research and the collection of more data are necessary.

\section{References}

Al-Ansari, M., Mokdad, M., 2009. Anthropometrics for the design of Bahraini schoo furniture. International Journal of Industrial Ergonomics 39, 728-735.

Barroso, M.P., Arezes, P.M., da Costa, L.G., Miguel, A.S., 2005. Anthropometric study of Portuguese workers. International Journal of Industrial Ergonomics 35 401-410.

Boussena, M., Davies, B.T., 1989. An adjustable vice mount. Applied Ergonomics 20 (2), 141-142.

Das, B., Wimpee, J., Das, B., 2002. Ergonomics evaluation and redesign of a hospital meal cart. Applied Ergonomics 33, 309-318.

Dewangan, K.N., Owary, C., Datta, R.K., 2008. Anthropometric data of female farm workers from north eastern India and design of hand tools of the hilly region. Industrial Journal of Industrial Ergonomics 38, 90-100.

Gonen, E., Kalinkara, V., Ozgen, O., 1991. Anthropometry of Turkish women. Technical Note. Applied Ergonomics 6, 409-411.

Huang, C., You, M., 1994. Anthropometry of Taiwanese women. Technical Note Applied Ergonomics 25, 186-187.

International Monetary Fund, 2008. World Economic Outlook Database [online] Available from: http://imf.org/external/pubs/ft/weo/2009/02/weodata/index. aspx (accessed 19.10.09).

İseri, A., Arslan, N., 2009. Estimated anthropometric measurements of Turkish adults and effects of age and geographical regions. International Journal of Industrial Ergonomics 39, 860-865.

Jung, H.S., 2005. A prototype of an adjustable table and an adjustable chair for schools. International Journal of Industrial Ergonomics 35, 955-969.

Klamklay, J., Sungkhapong, A., Yodpijit, N., Patterson, P.E., 2008. Anthropometry of the southern Thai population. International Journal of Industrial Ergonomics 38, $111-118$.

Kroemer, K.H.E., Grandjean, E., 1997. Fitting the Task to the Human: A Textbook of Occupational Ergonomics, fifth ed. CRC Press.

Kothiyal, K., Tettey, S., 2000. Anthropometry data of elderly people in Australia. Applied Ergonomics 20, 329-332.

Lim, L.E.N., Ngan, L.M.T., Lam, C.Y., 1990. Anthropometry research at NTI. HFE Seminar, Improving Human Performance and Well-Being.

Lin, Y.C., Wang, M.J.J., Wang, E.M., 2004. The comparisons of anthropometric characteristics among four peoples in East Asia. Applied Ergonomics 35, $173-178$.

Lohr, S.L., 1999. Sampling: Design and Analysis. Duxbury.

Mandahawi, N., Imrhan, S., Al-Shobaki, S., Sarder, B., 2008. Hand anthropometry survey for the Jordanian population. International Journal of Industrial Ergonomics 38 (11-12), 966-976.

Mououdi, M.A., 1997. Static anthropometric characteristics of Tehran University student 20-30. Technical Note. Applied Ergonomics 28 (2), 149-150.

Park, J., Nam, Y., Lee, E., Park, S., 2009. Error detection in three-dimensional surface anthropometric data. International Journal of Industrial Ergonomics 39 (1), 277-282.

Pheasant, S.T., 1982. A technique for estimating anthropometric data from the parameters of the distribution of stature. Ergonomics 25, 981-992.

Pheasant, S., Haslegrave, C.M., 2006. Body Space: Anthropometry, Ergonomics, and the Design of Work, third ed. Taylor \& Francis.

Sirajuddin, S.M., Duggirala, R., Crawford, M.H., 1994. Population structure of the Chenchu and other south Indian tribal groups: relationships between genetic anthropometric, dermatoglyphic, geographic, and linguistic distances. Human Biology: An International Record of Research 66 (5), 865-884.

Thariq, M.G.M., Munasinghe, H.P., Abeysekara, J.D., 2010. Designing chairs with mounted desktop for university students: ergonomics and comfort. International Journal of Industrial Ergonomics 40, 8-18.

Wall, H.A.D., 1993. Environmental factors influencing growth and pubertal development. Environmental Health Perspectives Supplements 101 (2), 39-44.

Wang, E.M., Chao, W.C., 2010. In searching for constant body ratio benchmarks. International Journal of Industrial Ergonomics 40 (1), 59-67.

World Health Organization (WHO), 2008. Body Mass Index (BMI) Classification [Online] Available from: http://apps.who.int/bmi/index.jsp?introPage=intro_3. html (accessed 04.11.08). 